\title{
Patient safety in emergency medical services: executive summary and recommendations from the Niagara Summit
}

\author{
Blair L. Bigham, MSc, ACPf*; Ellen Bull, BScN, MAEd ${ }^{\dagger}$; Merideth Morrison, ACP $^{\ddagger}$;

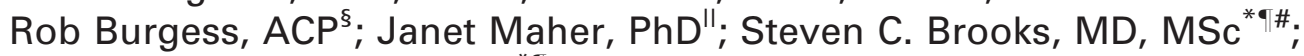 \\ Laurie J. Morrison, MD, MSc ${ }^{*}$ on behalf of the Pan-Canadian Patient Safety in EMS Advisory Group
}

\section{ABSTRACT}

Emergency medical services (EMS) personnel care for patients in challenging and dynamic environments that may contribute to an increased risk for adverse events. However, little is known about the risks to patient safety in the EMS setting. To address this knowledge gap, we conducted a systematic review of the literature, including nonrandomized, noncontrolled studies, conducted qualitative interviews of key informants, and, with the assistance of a pan-Canadian advisory board, hosted a 1-day summit of 52 experts in the field of EMS patient safety. The intent of the summit was to review available research, discuss the issues affecting prehospital patient safety, and discuss interventions that might improve the safety of the EMS industry. The primary objective was to define the strategic goals for improving patient safety in EMS. Participants represented all geographic regions of Canada and included administrators, educators, physicians, researchers, and patient safety experts. Data were collected through electronic voting and qualitative analysis of the discussions. The group reached consensus on nine recommendations to increase awareness, reduce adverse events, and suggest research and educational directions in EMS patient safety: increasing awareness of patient safety principles, improving adverse event reporting through creating nonpunitive reporting systems, supporting paramedic clinical decision making through improved research and education, policy changes, using flexible algorithms, adopting patient safety strategies from other disciplines, increasing funding for research in patient safety, salary support for paramedic researchers, and access to graduate training in prehospital research.

\section{RÉSUMÉ}

Le person-nel des services médicaux d'urgence (SMU) doit souvent intervenir dans des contextes difficiles et instables pouvant accroître les risques d'événements indésirables. Or, les risques pour la sécurité des patients dans ce contexte sont méconnus. Pour remédier à cette situation, nous avons effectué une analyse documentaire complète, y compris des études non randomisées et non contrôlées, réalisé des entrevues qualitatives d'informateurs clés et, avec l'assistance d'un conseil consultatif pancanadien, organisé une table ronde d'une journée réunissant 52 experts dans le domaine de la sécurité des patients et des SMU. Le but de cette rencontre était d'examiner les recherches disponibles, de discuter des questions touchant la sécurité des patients en milieu préhospitalier et de discuter des interventions pour améliorer leur sécurité. Notre principale intention était de définir les objectifs stratégiques pour améliorer la sécurité des patients dans les SMU. Les participants représentaient toutes les régions géographiques du Canada et incluaient des administrateurs, des éducateurs, des médecins, des chercheurs et des experts en sécurité des patients. Les données ont été recueillies à l'aide d'un vote électronique et $d^{\prime}$ 'analyses qualitatives des discussions. Le groupe d'experts est parvenu à un consensus sur 9 recommandations pour accroître la sensibilisation, réduire les événements indésirables et proposer des orientations de recherche et d'apprentissage relatives à la sécurité des patients dans les SMU : prise de conscience croissante des principes de la sécurité des patients, amélioration de la déclaration des événements indésirables par la création de systèmes de signalement non punitif, appui de la prise de décision clinique par les

From the *Rescu, Keenan Research Centre, Li Ka Shing Knowledge Institute, St. Michael's Hospital, University of Toronto, Toronto, ON; †School of Community and Health Studies, Centennial College, Toronto, ON; ¥Simcoe County Paramedic Services, Barrie, ON; §Sunnybrook-Osler Centre for Prehospital Care, Toronto, ON; IIApplied Research and Innovation Centre, Centennial College, Toronto, ON; ๆDepartment of Medicine, Division of Emergency Medicine, University of Toronto, Toronto, ON; and \#Department of Emergency Services, Sunnybrook Health Sciences Centre, Toronto, ON.

Correspondence to: Blair Bigham, BSc MSc ACPf, Rescu, Keenan Research Centre, Li Ka Shing Knowledge Institute, St. Michael's Hospital and University of Toronto, 30 Bond Street, Toronto, ON M5B 1W8; bighamb@smh.toronto.on.ca.

This article has been peer reviewed. 
paramédics par l'amélioration de la recherche et de la formation, modifications des politiques, utilisation d'algorithmes flexibles, adoption de stratégies de sécurité des patients provenant d'autres disciplines, augmentation du financement pour la recherche en sécurité des patients, aide salariale pour les chercheurs paramédicaux, et accès à une formation postdoctorale en recherche relative aux soins préhospitaliers.

Keywords: adverse events, emergency medical services, paramedicine, patient safety, safety culture
Emergency medical services (EMS) personnel care for patients in challenging and dynamic environments that may contribute to an increased risk for adverse events. However, little is known about the risks to patient safety in the EMS setting. To address this knowledge gap, the Canadian Patient Safety Institute (CPSI) partnered with the Emergency Medical Services Chiefs of Canada (EMSCC) and the Calgary EMS Foundation to fund research examining patient safety in the EMS setting. The phases of the research involved a systematic review of the literature, qualitative interviews of key informants from Canada and abroad, and a 1-day summit that brought together leaders in EMS and patient safety experts to discuss the successes, challenges, and future directions of the patient safety movement in Canadian prehospital care. ${ }^{1}$

The first Canadian Patient Safety in EMS Summit was held June 1, 2009, in Niagara Falls, Ontario, and included 52 patient safety, EMS, and research experts, 51 from across Canada and 1 from the United States. The definition of patient safety employed by the World Health Organization (WHO) was used where patient safety is defined as the "reduction of risk of unnecessary harm associated with healthcare to an acceptable minimum." "2 The term "acceptable minimum" refers to the collective notions given current knowledge, the resources available, and the context in which care was delivered weighed against the risk of nontreatment or other treatment. ${ }^{1}$ Results from the systematic review and key informant interviews were presented to the participants, who were then encouraged to read, analyze, and discuss the results and share their own beliefs in both small groups and collectively. Information that emerged through discussion was captured using both anonymous electronic responses and note taking by the research team, which was subsequently thematically analyzed. Three breakout sessions chaired by an external professional facilitator fostered discussion on reactions to the research findings and led to the identification of innovative opportunities, existing programs and tools applicable to EMS patient safety, and next steps to address patient safety challenges in Canadian EMS. This document is an executive summary of that day's dynamic, wideranging discussions. Our hope is that the consensus we present may serve to guide future research and policy relating to prehospital patient safety.

\section{PARTICIPANTS}

The participants were selected by a pan-Canadian advisory committee and identified their current primary role in EMS or a related field as patient safety experts $(8 \%)$, EMS administrators $(61 \%)$, physicians $(16 \%)$, other health care professionals $(5 \%)$, educators $(3 \%)$, or other $(8 \%)$. None of the participants were practicing paramedics $(0 \%)$; however, many were credentialed and previously active in the field. Two-thirds of participants reported having $>20$ years of experience in their respective fields. Participants were not compensated or reimbursed for any costs associated with the summit.

\section{ISSUES}

The discussion was grounded on issues identified in a systematic review of the world's literature on EMS patient safety that included noncontrolled trials and qualitative studies. In addition, the participants reviewed the results of a qualitative study of key informants who were interviewed to identify the central issues related to patient safety in EMS in the United States and Canada. ${ }^{3}$ Summit discussants were asked to rate the importance and feasibility of the themes that emerged from the systematic review and qualitative research on 5-point Likert scales (anchored by $1=$ not important, $5=$ very important or $1=$ not feasible to implement, 5 = very feasible to implement as appropriate) using hand-held technology (Table 1). Scores of 4 and 5 were combined to identify issues of high importance or feasibility.

The most prominent patient safety issue discussed was clinical judgment and decision making, rated as highly important by $95 \%$ of attendees. There was a 


\begin{tabular}{|c|c|c|c|c|c|c|c|c|c|c|}
\hline \multirow[b]{2}{*}{ Theme } & \multicolumn{5}{|c|}{ Importance (\%) } & \multicolumn{5}{|c|}{ Feasibility (\%) } \\
\hline & 1 & 2 & 3 & 4 & 5 & 1 & 2 & 3 & 4 & 5 \\
\hline Clinical judgment and training & 0 & 3 & 3 & 11 & 84 & 0 & 5 & 26 & 34 & 32 \\
\hline Medication adverse events & 0 & 13 & 18 & 28 & 41 & 0 & 5 & 31 & 36 & 28 \\
\hline Intubation & 0 & 13 & 26 & 26 & 36 & 0 & 11 & 18 & 39 & 29 \\
\hline Vehicle collisions & 3 & 13 & 23 & 33 & 28 & 0 & 0 & 13 & 33 & 54 \\
\hline \multicolumn{10}{|c|}{ Relationship of EMS to the health care system } & 13 \\
\hline Interfacility transport & 3 & 26 & 16 & 29 & 26 & 3 & 10 & 31 & 31 & 23 \\
\hline Aircraft safety & 10 & 26 & 18 & 31 & 10 & 8 & 21 & 8 & 28 & 33 \\
\hline
\end{tabular}

consensus that paramedics in Canada are providing increasingly complex and time-sensitive patient care; examples include new cardiopulmonary resuscitation (CPR) process measures for cardiac arrest, ST elevation myocardial infarction identification and transport bypass protocols, early stroke identification and transport bypass protocols, and therapeutic interventions in trauma. It was felt that training may be inadequate to ensure that paramedics are competent in performing complex protocols and making clinical decisions regarding diagnosis and treatment, and this may contribute to patient safety issues. Alternatively, it was also felt that paramedics are occasionally penalized for exercising good clinical judgment under the current model of protocol-driven care and can face disciplinary actions in response to protocol deviations. Although the importance of protocols was recognized, they were viewed as a "double-edged sword," and some suggested that guidelines incorporating flexibility to include clinical decisions and judgment may be more appropriate if coupled with timely medical oversight, comprehensive training, and feedback. There was a consensus that a culture of fear exists in EMS services as a result of aggressive disciplinary action, a delay before peer and medical review, and an emphasis on finding fault rather than a balanced systems approach to feedback and identifying areas of weaknesses. This culture of fear may jeopardize accurate reporting of adverse events. Sixty-six percent of participants indicated that the feasibility of implementing a cultural shift was high.

Medication adverse events were rated the second most important patient safety issue, with 69\% of participants rating the importance of this highly. The perception of participants was that events of this nature caused few prehospital deaths; however, we were unable to identify literature reporting a death rate attributed to medication error. Sixty-four percent of participants rated medical adverse events feasible to address. Participants agreed that the scope of medication adverse events, a scope that includes errors of both commission and omission, is poorly understood because no standardized definitions or data collection systems exist. Here, too, it was felt that a culture of fear compromises our ability to define incidence. Participants noted that EMS staff may not divulge medication adverse events for fear of disciplinary action or peer ostracization. The identification of system causes of adverse events, rather than addressing individual competency, was emphasized as a beneficial strategy. Medication label similarities resulting in identification errors at the point of care were noted to be an example of adverse events attributable to system factors.

It was felt that medication adverse events could be reduced through the use of appropriate and validated tools to support clinical decision making, such as dosing charts and unit dose supplies. Participants felt that a standardized national reporting structure should be implemented and that the punitive culture aimed at identifying individuals must change to encourage disclosure of both "near misses" and adverse events. Finally, it was felt that standardized systems to log, label, distribute, stock, and store medications currently in use in other disciplines could reduce systemic causes of adverse events.

Despite the substantial body of research on the safety of paramedic intubation, ${ }^{4-13}$ there was skepticism 
among participants that intubation failure contributes to a significant proportion of adverse outcomes. Unfortunately, our current data are unable to resolve this issue. Wang and colleagues used a populationbased data set in an effort to identify the shortcomings of documentation with respect to intubation and our subsequent inability to comment on adverse events related to advanced airway techniques. ${ }^{13,14}$ What remains lacking is a common set of definitions and reporting on intubation attempts, successes, and failures. Sixty-two percent of participants rated intubation as an important issue, and $68 \%$ felt that it was feasible to address. The consensus suggested that skills and decisions concerning intubation may be enhanced through human patient simulators, extended residency and preceptorship, and additional clinical time in the operating room setting. It appears that a need exists in the area of airway management to evaluate new procedures and treatments and to reevaluate the efficacy, education, and risk involved with established procedures and treatments from the perspective of patient safety. Little is known about adjunctive airway skills, and further research into patient safety for interventions such as supraglottic airways and timing systems to avoid hyperventilation would be helpful.

The final major theme that was discussed at the summit was ground ambulance collisions. Many participants recounted anecdotes regarding vehicle collisions, but none were aware of a national database that tracked the incidence of these events. Previously identified causes of collisions included the use of lights and siren responses, inexperienced staff, the effects of shift work and stress in EMS, and a lack of driver safety training. ${ }^{15-17}$ Sixty-one percent of participants rated collisions as an important issue, and it was considered by $87 \%$ to be feasible to address. Ground ambulance collisions can be prevented by avoiding unwarranted lights and siren responses through the use of validated commercial dispatch software. ${ }^{18}$ The use of speed monitoring programs using tachometers was noted to have been successfully implemented in a number of EMS services and was felt to reduce risky driving behaviour.

Other patient safety initiatives identified prior to the discussions (including the relationship of EMS to the health care system, aircraft safety, and interfacility transport) were deemed by the participants to be less significant or feasible to change and were not addressed in detail.
Table 2. Canadian Patient Safety Competencies

Domain 1: Contribute to a culture of patient safety

A commitment to applying core patient safety knowledge, skills, and attitudes to everyday work

Domain 2: Work in teams for patient safety

Working within interprofessional teams to optimize both patient safety and quality of care

Domain 3: Communicate effectively for patient safety

Promoting patient safety through effective health care communication

Domain 4: Manage safety risks

Anticipating, recognizing, and managing situations that place patients at risk

Domain 5: Optimize human and environmental factors

Managing the relationship between individual and environmental characteristics to optimize patient safety

Domain 6: Recognize, respond to, and disclose adverse events Recognizing the occurrence of an adverse event or close call and responding effectively to mitigate harm to the patient, encourage disclosure, and prevent recurrence

Adapted from The Safety Competencies Steering Committee. The Safety

Competencies: enhancing patient safety across the health professions. Ottawa:

Canadian Patient Safety Institute; 2008. Available at: www.patientsafetyinstitute.ca.

\section{IDENTIFYING THE GAPS AND POTENTIAL SOLUTIONS}

There was much discussion by the summit participants on the ambiguity of the role of the paramedic in the health system and the alignment of the practice of paramedicine with either public safety services (particularly police and fire) or health care services. In Canada, paramedic competencies are defined by the Paramedic Association of Canada in the National Occupation Competency Profile, ${ }^{19}$ and credentialing requirements are well established and similar to those of other health care professions, with postsecondary education programs accredited by the Canadian Medical Association. After significant debate, the consensus opinion supported the paradigm of paramedicine as a health care profession. The Canadian patient safety competencies published by the CPSI review six important patient safety principles for health care professionals, ${ }^{1}$ and participants favoured including these in the competency profile ${ }^{19}$ to ensure that all paramedics receive this training (Table 2).

A call for further high-quality research in patient safety resonated with the participants, and the lack of human resource capacity in EMS to conduct this research was identified as a substantial barrier requiring attention. Paramedicine is a growing profession, and high-quality research is becoming more common in the field. Community colleges are beginning to initiate 
applied research as an element in paramedic training curricula, and the number of paramedics with bachelor's degrees or graduate-level training is increasing. An evolution toward a bachelor's degree in paramedicine as the educational preferred route into the profession and graduate training in paramedicine were identified as key steps in building human capacity to studying patient safety and implementing evidence-based changes. Graduate training in research and administrative leadership may improve data capture through heightened appreciation of its value and promote decision making based on reliable and valid data.

Participants felt that a focus on graduate training should be encouraged through staff tuition support and dedicated time while employed in an EMS service. Models such as the Ontario Graduate Scholarship for EMS research at the University of Toronto could be replicated elsewhere. The University of Toronto scholarship is matched 2 for 1 by an investment from the province's Emergency Health Services Branch of the Ministry of Health and Long-Term Care to provide salary support for individuals pursuing EMS research. This scholarship is open to any graduate student in the Faculty of Medicine, including allied health professionals (paramedicine). Dalhousie University has a similar partnership program with the government of Nova Scotia. The Heart and Stroke Foundation recently revised their eligibility criteria for scholarship awards to include paramedics pursuing graduate training or postdoctoral training as a research fellow. ${ }^{20}$

Improving clinical judgment requires optimum understanding of the cognitive process in making decisions. One way to address this is to enhance education delivery and retention in paramedic graduates. Other tools include morbidity and mortality rounds and root cause analysis, increasing the use of simulation in initial and ongoing education, and increasing interdisciplinary training. Summit participants felt that encouraging paramedic educators to pursue graduate degrees in education would build capacity, enhance education delivery, and lead to evidence-based teaching methodology.

\section{STRATEGIC PRIORITIES}

Participants identified and reached consensus on nine strategic priorities to improve patient safety in EMS:

1. Make patient safety a corporate value within EMS organizations and the profession.
2. Include patient safety domains identified in the safety competencies ${ }^{1}$ in the National Occupational Competency Profile ${ }^{19}$ for paramedic trainees and in ongoing continuing medical education sessions.

3. Create a Web-based reporting system for adverse events unique to the prehospital setting.

4. Increase funding for patient safety and operations research and increase access to salary support for those with research expertise.

5. Create or contribute to the development of universal definitions, indicators, and outcomes relating to patient safety in EMS.

6. Support through advocacy, research, and operational structure the concept that paramedics are capable of decision making and judgment and create more flexible algorithms that allow clinical judgment to be exercised.

7. Examine the literature from other disciplines with similarities to EMS for patient safety interventions that could be applied directly or with slight modification to the prehospital setting.

8. Support advanced training in EMS research, education, and patient safety expertise to build human resource capacity. Examples of support that have worked in other disciplines include salary support, reduced clinical hours, tuition support, and scholarships.

9. Promote the identification and reporting of highrisk activities performed by paramedics through a culture of support and engagement of the providers without fear of punishment to focus attention on system issues rather than individuals.

\section{LIMITATIONS}

Our findings are limited by several factors. First, the lack of involvement by field paramedics limits the perspective of the discussants. Several paramedic invitees reported an inability to secure travel funding or time off work to attend the summit and were unable to accept invitations to participate. This was partly attributed to the economic climate at the time of the summit. Second, the lack of data and uniform definitions supporting and defining the recommendations is important. The recommendations are based on a paucity of evidence identified through the systematic review and interviews of key informants and reflect consensus opinion. The discussions were limited to patient safety and did not address provider safety; however, EMS administrators felt strongly that 
provider safety also requires more attention and research. Finally, there is the potential for bias; only $60 \%$ of invited participants accepted the invitation to attend. Despite these limitations, and given the lack of attention to date in patient safety in EMS, this summit and our findings represent an important first step in addressing patient safety in prehospital care.

\section{CONCLUSION}

The Patient Safety in EMS Summit identified current issues and areas of focus related to prehospital patient safety in Canada and provided strategic priorities to improve safety in many areas of EMS care. A review of the literature identified a paucity of research related to patient safety in EMS and qualitative work, and discussion at this summit revealed knowledge gaps regarding important aspects of patient safety, including clinical decision making. Summit participants felt that investing in people willing to pursue patient safety research will generate a body of knowledge that can be applied to the EMS industry to advance the practice of paramedicine to benefit systems, providers, and patients.

Acknowledgements: This summit was funded by the CPSI, the EMSCC, and the Calgary EMS Foundation. Collection of all summary statements and data was completed by the research team, and Janet Dalicandro of Enrichment Enterprises Inc. was hired to facilitate the summit. The Pan-Canadian Patient Safety in EMS Advisory Group played a key role in moderating the group discussions. This group was chaired by Joe Acker, EMSCC Board of Directors, and included Dr. Andy Anton, Paula Beard, Ian Blanchard, Ron Bowles, Dr. Ken Bucholz, Pierre Deschamps, Orvie Dingwall, Tom Dobson, Paula Greco, Lyle Karasiuk, Sandi Kossey, John Lewis, Dr. Russell MacDonald, Marie Owen, Dr. Brian Schwartz, Bryan Singleton, and Jennifer Wheaton. More information about this advisory group can be found on the CPSI website at <http:// www.patientsafetyinstitute.ca $>$. Sandi Kossey, from the CPSI, provided additional support and guidance.

Competing interests: None declared.

\section{REFERENCES}

1. Bigham BL, Brooks SC, Maher J, et al. Patient safety in emergency medical services: advancing and aligning the culture of patient safety in EMS. Canadian Patient Safety Institute: Edmonton, Alberta; 2009. Available at: http://www.patientsafetyinstitute.ca/English/ Initiatives/EmergencyMedicalServices/Pages/default.aspx (accessed June 29, 2010).

2. World Health Organization. WHO international classification for patient safety. Available at: http://www.who.int/patientsafety/ implementation/taxonomy/en/ (accessed December 2, 2009).
3. Atack L, Maher J. Emergency medical and health providers' perceptions of key issues in prehospital patient safety. Prehosp Emerg Care 2010;14:95-102.

4. Bair AE, Smith D, Lichty L. Intubation confirmation techniques associated with unrecognized non-tracheal intubations by pre-hospital providers. 7 Emerg Med 2005;28:403-7.

5. DiRusso TM, Sullivan T, Risucci D, et al. Intubation of pediatric trauma patients in the field: predictor of negative outcome despite risk stratification. 7 Trauma 2005;59:84-90.

6. Fakhry SM, Scanlon JM, Robinson L, et al. Prehospital rapid sequence intubation for head trauma: conditions for a successful program. 7 Trauma 2006;60:997-1001.

7. Jemmett ME, Kendal KM, Fourre MW, et al. Unrecognized misplacement of endotracheal tubes in a mixed urban to rural emergency medical services setting. Acad Emerg Med 2003;10:961-5.

8. Jones JH, Murphy MP, Dickson RL, et al. Emergency physician-verified out-of-hospital intubation: miss rates by paramedics. Acad Emerg Med 2004;11:707-9.

9. Newton A, Ratchford A, Khan I. Incidence of adverse events during prehospital rapid sequence intubation: a review of one year on the London Helicopter Emergency Medical Service. 7 Trauma 2008;64:487-92.

10. Parwani V, Hoffman RJ, Russell A, et al. Practicing paramedics cannot generate or estimate safe endotracheal tube cuff pressure using standard techniques. Prebosp Emerg Care 2007;11:307-11.

11. Pratt JC, Hirshberg AJ. Endotracheal tube placement by EMT-basics in a rural EMS system. Prehosp Emerg Care 2005;9:172-5.

12. Svenson JE, Lindsay MB, O'Connor JE. Endotracheal intracuff pressures in the ED and prehospital setting: is there a problem? Am 7 Emerg Med 2007;25:53-6.

13. Wang HE, Lave JR, Sirio CA, et al. Paramedic intubation errors: isolated events or symptoms of larger problems? Health Affairs 2006;25:501-9.

14. Wang HE, Kupas DF, Paris PM, et al. Failed prehospital intubations: an analysis of emergency department courses and outcomes. Prehosp Emerg Care 2001;5:134-41.

15. Kahn CA, Pirrallo RG, Kuhn EM. Characteristics of fatal ambulance crashes in the United States: an 11-year retrospective analysis. Prehosp Emerg Care 2001;5:261-9.

16. Saunders CE, Heye CJ. Ambulance collisions in an urban environment. Prehosp Disaster Med 194; 9:118-24.

17. Wang HE, Fairbanks RJ, Shah MN, et al. Tort claims and adverse events in emergency medical services. Ann Emerg Med 2008;52:256-62.

18. Medical Priority Dispatch System. August 21, 2009. Available at: www.medicalpriority.com (accessed December 2, 2009).

19. Paramedic Association of Canada. National Occupational Competency Profile. Available at: http://www.paramedic.ca/ Content.aspx?ContentID $=4 \&$ Content TypeID $=2$ (accessed December 2, 2009).

20. Heart and Stroke Foundation of Canada. Fump Start Resuscitation. 2009. Available at: http://www.hsf.ca/research/ en/strategic-funding-opportunities/jump-start-resuscitation2009.html (accessed December 2, 2009). 